\title{
Elective Discontinuation of CDK4/6 Inhibitors in Patients with Metastatic Hormone Receptor- Positive, Her-2-Negative Breast Cancer: A Retrospective Single-Center Experience
}

\author{
Thomas Decker $^{\mathrm{a}} \quad$ Robert Seifert $^{\mathrm{b}} \quad$ Matthias Bichler $^{\mathrm{a}} \quad$ Andrea Birtel $^{\mathrm{a}}$ \\ Gerhard Fischer $^{\mathrm{a}, \mathrm{c}}$ Christoph Nonnenbroich ${ }^{\mathrm{a}}$ Tobias Dechow $^{\mathrm{a}}$ \\ ${ }^{a}$ Onkologie Ravensburg, Ravensburg, Germany; ${ }^{b}$ Department of Nuclear Medicine, University Hospital Essen, Essen, \\ Germany; 'St. Elisabeth Hospital, Ravensburg, Germany
}

\section{Keywords}

Breast cancer - Cyclin-dependent 4/6 kinase therapy . Discontinuation

\begin{abstract}
Introduction: Cyclin-dependent 4/6 kinase (CDK4/6) inhibitors given with endocrine therapy until disease progression are standard of care in the treatment of women with advanced HR-positive Her-2-negative breast cancer. No data are available if therapy can be safely de-escalated to endocrine monotherapy in patients with long-lasting disease control. Methods: We performed a retrospective analysis on the clinical course of 22 patients at our center who received CDK4/6 inhibitors with aromatase inhibitors or fulvestrant. All patients had at least stable disease for $>6$ months and made a joint decision with their provider to electively discontinue CDK4/6 inhibitors. Best objective response (BOR) at treatment discontinuation, progression-free survival, and retreatment characteristics were recorded. Results: Of 138 patients who received CDK4/6 inhibitors as first- or second-line therapy at our center, 22 met the inclusion criteria. Median duration of CDK4/6 treatment was 18 months (range 6-45). BOR was complete response in 1, partial response in 8 , and stable disease in 13 patients. After a median duration of endocrine monotherapy of 9.5 months (range 5-44 months), 6 of 22 patients had progressive disease ( 1 local relapse and 5 systemic progression). All patients with disease progression had at least stable disease to chemotherapy $(N=1)$ or re-
\end{abstract}

treatment with CDK4/6 inhibitors $(N=4)$. Conclusion: Elective discontinuation of CDK4/6 inhibitors is feasible in patients with long-lasting disease stabilization. This strategy should be evaluated in prospective trials.

๑) 2021 S. Karger AG, Basel

\section{Introduction}

CDK4/6 inhibitors given with endocrine treatment (CE treatment) have changed the treatment landscape in patients with metastatic HR-positive/Her-2-negative breast cancer. This treatment modality has largely replaced chemotherapy or endocrine monotherapy as the first-line treatment even in patients with visceral disease [1].

The combination of endocrine treatment with a CDK4/6 inhibitor abemaciclib (A), palbociclib (P), or ribociclib ( $\mathrm{R}$ ) has demonstrated to be more effective than endocrine monotherapy in first-line or second-line treatment resulting in impressive improvements in progression-free survival (PFS) [2,3] and overall survival (OS) [4, 5]. In addition, $\mathrm{CE}$ treatment has been shown to be at least as effective as chemotherapy in the first/second-line setting $[6,7]$. Although serious adverse events are rare in patients treated with either drug, substantial chronic toxicities are common and can interfere with quality of life. In the case of $\mathrm{P}$ and $\mathrm{R}$, chronic immunosuppression by long-lasting neutropenia is also a concern [8]. 
Table 1. Patient characteristics and comparison of patients with and without DP

\begin{tabular}{lllll}
\hline Characteristics & Value & DP & No DP & All patients \\
\hline Patients & $N$ & 5 & 17 & 22 \\
Age & Median (range) & $78(54-85)$ & $77(57-89)$ & $78(54-89)$ \\
Visceral disease & n/total & $1 / 5$ & $9 / 17$ & $10 / 22$ \\
CDK4/6 inhibitor & A/P/R, & $0 / 3 / 2$ & $3 / 6 / 8$ & $3 / 9 / 10$ \\
BOR & SD/CR + PR & $3 / 2$ & $10 / 7$ & $13 / 9$ \\
Length of CE treatment & Months, median (range) & $22(16-40)$ & $15(6-44)$ & $18(6-44)$ \\
Length of endocrine monotherapy & Months, median (range) & $7.7(1.5-16)$ & $10(6.5-44.5)$ & $9.5(1.5-44.5)$ \\
\hline
\end{tabular}

$\mathrm{DP}$, disease progression; $\mathrm{CE}, \mathrm{CDK} 4 / 6$ inhibitors with endocrine treatment; $\mathrm{BOR}$, best objective response with CE; A, abemaciclib; P, palbociclib; R, ribociclib.

The duration of treatment with CDK4/6 inhibitors needed to obtain PFS or OS benefits is not known, but prolonged cell cycle arrest might contribute to the development of resistance by acquired mechanisms [9]. Although endocrine treatment used as maintenance therapy after chemotherapy $[10,11]$ in patients with metastatic breast cancer does not seem to be effective, endocrine monotherapy can result in long-term disease control when given in the first-line setting - especially in patients with bone only disease [12]. In the current work, we have conducted a retrospective analysis of patients who have electively discontinued CDK4/6 inhibitor treatment in a joint decision with their treatment provider while maintaining endocrine monotherapy.

\section{Methods}

After ethics board approval (Landesärztekammer Baden Württemberg, F-2021-014), data of patients who started CE treatment at the Onkologie Ravensburg from November 18, 2016 (the approval date of pabociclib) to December 30, 2020, were retrieved from the electronic medical records. Inclusion criteria included a pathology-confirmed diagnosis of a HR-positive Her-2-negative metastatic breast cancer, disease control (stable disease, partial remission, and complete remission) on treatment with CE as firstline or second-line treatment for at least 6 months, and the presence of a joint decision of the patient and the treating oncologist to discontinue CDK4/6 inhibitors in the absence of severe toxicities. Written informed consent was obtained from all patients participating in the study. Patient demographics, tumor characteristics, treatment history, and efficacy data (BOR, PFS, and re-treatment data) were collected. PFS was computed from the date of last CDK4/6 inhibitor treatment to the date of progression.

\section{Results}

\section{Patient Characteristics}

Detailed patient characteristics are provided in $\mathrm{Ta}$ ble 1 . Of 138 patients who started treatment with $\mathrm{CE}$ as first or second line, 22 patients met the inclusion criteria.
Median age was 77 years, and 20/22 patients were postmenopausal. All patients received a combination of aromatase inhibitor (20) or fulvestrant (2) and a CDK4/6 inhibitor (A: 3, P: 9, and R: 10) (2 premenopausal patients in combination with GNRH analog). Eleven of 22 patients had bone only disease, and most patients were treated in the first-line setting (20/22). One patient had a $\mathrm{CR}$ as BOR before CDK4/6 inhibitor de-escalation, 8 had PR, and 13 had SD. Median duration of CE was 18 months (range 6-44 months). Thirteen patients had de novo metastatic disease at the beginning of $\mathrm{CE}$ treatment, and 4 patients had endocrine resistance as defined by $\mathrm{ABC}$ consensus guidelines [13].

\section{Treatment and Response}

After a median observation time of 9.5 months from the last dose of CDK4/6 inhibitor, 15/22 patients still had no signs of disease progression at the last follow-up. One patient with bone only disease had rapid disease progression 1.5 months after the CDK4/6 inhibitor was discontinued with newly detectable liver metastasis. This patient is currently in remission again after chemotherapy with paclitaxel and bevacizumab was started. Four patients (one with visceral disease) had disease progression 5, 8, 15, and 16 months after last CDK4/6 inhibitor treatment. These patients had disease progression with bone metastasis (2 patients), cutaneous (1 patient), and lymphonodal (1 patient) disease. All patients subsequently had ongoing disease control with reintroduction of CE treatment (3 SD and 1 PR for 4, 5, 6, and 6 months, respectively). Endocrine treatment was unchanged in 3 patients and changed from fulvestrant to exemestane in 1 patient. One patient with ongoing endocrine monotherapy had a local relapse (triple-negative histology) which was completely excised. One patient died due to unrelated cardiovascular disease 10 months after de-escalating treatment without signs of disease progression. Figure 1 summarizes the results in a swimmer blot showing length of $\mathrm{CE}, \mathrm{BOR}$, length of endocrine monotherapy, and re-treatment outcome. 
Fig. 1. A swimmer plot of all patients showing length of $\mathrm{CE}$ treatment, endocrine monotherapy, BOR, and re-treatment characteristics. BOR, best objective response.

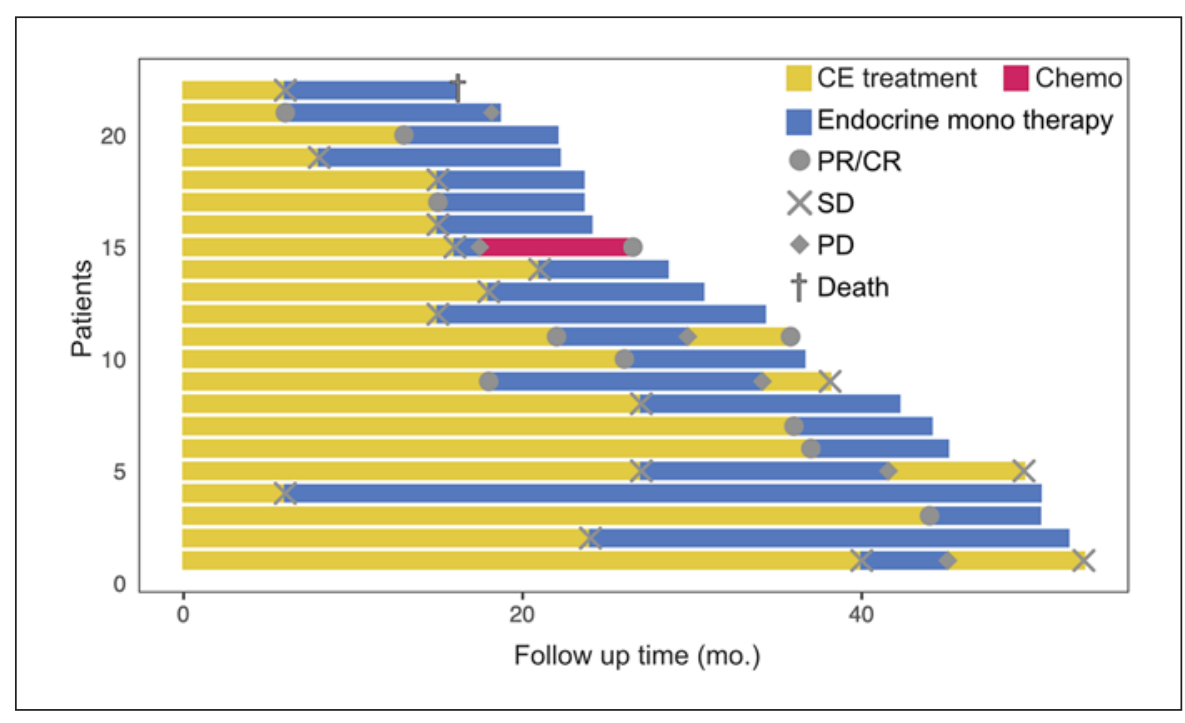

Disease progression was seen in 4 of 13 patients with SD as BOR and in 1 of 9 patients with PR/CR. Four out of 11 patients with a CE of 18 months or more had PD and 2 of 11 patients with a shorter CE duration. CE treatment was longer in patients with $\mathrm{PD}$ than in non-PD patients (22 vs. 15 months). Five of 12 patients with nonvisceral disease (11 with bone only and 1 with cutaneous disease) had PD compared to only 1 patient with visceral metastasis. These results are depicted in Table 1. None of these differences reached statistical significance (Fisher's exact test or Mann-Whitney test as appropriate, data not shown).

\section{Discussion}

CE treatment is a very effective treatment strategy in patients with metastatic HR-positive Her-2-negative breast cancer and is considered to be the standard of care as the first-line treatment. However, the optimal treatment duration is not well established.

To our knowledge, our retrospective study is the first to present data on the clinical course of patients who made the informed decision to electively de-escalate CE. Treatment de-escalation after disease stabilization is a common treatment strategy in a variety of cancers including breast cancer treated with chemotherapy [14-16], with checkpoint inhibitors [17], or with targeted therapies $[18,19]$.

In contrast, $\mathrm{CE}$ treatment is usually given until disease progression or the occurrence of intolerable toxicities. Adverse events differ between $\mathrm{P} / \mathrm{R}$ and A especially with regard to neutropenia and gastrointestinal toxicities. Although these adverse events are rarely severe, chronic long-term toxicities can interfere with quality of life [8].
Treatment visits are more frequent with CE treatment, add to treatment burden, and are of special concern in the current COVID-19 pandemic [20, 21]. Polypharmacy is another frequent problem in elderly cancer patients with a high potential for drug-drug interactions caused by CDK4/6 inhibitors [22]. In addition, despite its established benefits, the cost effectiveness of CE treatment is controversial in different health systems [23,24].

In our cohort of patients, $15 / 22$ patients were still progression free after a median time of endocrine monotherapy of 9.5 months. These data compare favorably with follow-up data from Paloma-2 and Monaleesa-2 which showed that barely $70 \%$ of patients who were progression free after 18 months of CE treatment were still without progression after 12 additional months of CE therapy [25, 26].

In our retrospective study, PFS events occurred in patients independent of the duration of CE or BOR (SD vs. $\mathrm{CR} / \mathrm{PR}$ ). Surprisingly, disease progression was more frequent in patients without visceral metastasis. This might be explained by the fact that $8 / 10$ patients with visceral disease had a PR as best response as compared to $11 / 12$ patients with SD and 1 patient with cutaneous metastasis with nonvisceral metastasis.

Our promising results demonstrate that drug holidays are feasible in the setting of CE treatment. Importantly, all patients with $\mathrm{PD}$ had subsequent disease control with reintroduction of CE treatment $(n=4)$ or chemotherapy $(n=1)$.

Withdrawal of targeted treatment has been shown to reverse acquired resistance in experimental systems [27]. Drug holidays could therefore lead to longer disease control with CE treatment interrupted by drug holidays. One patient had early and rapid disease progression immediately after cessation of CDK4/6 treatment and therefore 
received chemotherapy (with a good response) demonstrating that close follow-up of these patients is needed.

Our study faces some limitations. The patient cohort is relatively small, and selection bias is an important issue in retrospective studies. In addition, no data on quality of life, patient-reported outcomes, treatment burden, or adverse events were collected due to the retrospective nature of our study. However, the promising results of our retrospective patient series warrant evaluation of treatment de-escalation in patients with prolonged disease stabilization in prospective clinical trials.

\section{Conclusion}

Discontinuation of CDK4/6 inhibitors seems to be safe in selected patients with metastatic HR-positive Her2-negative breast cancer and prolonged disease control. This strategy should be further explored in randomized trials.

\section{Statement of Ethics}

This study was conducted as a retrospective analysis of patient data and was approved by the Landesärztekammer Baden Württemberg (Reference No. F-2021-014). Written informed consent was obtained from all patients. This research project complied with the guidelines for human studies and was conducted ethically in accordance with the World Medical Association Declaration of Helsinki.

\section{Conflict of Interest Statement}

Thomas Decker received honoraria from Iomedico and advisory board honoraria from Novartis and Roche. Tobias Dechow received honoraria from Astra Zeneca, Boehringer Ingelheim, Iomedico, Merck, and Sanofi. Gerhard Fischer received advisory board honoraria from BMS and Roche.

\section{Funding Sources}

This research did not receive grants from any funding agency in the public, commercial, or not-for-profit sectors.

\section{Author Contributions}

Thomas Decker and Tobias Dechow contributed to conception and design. Thomas Decker, Tobias Dechow, Matthias Bichler, Andrea Birtel, and Christoph Nonnenbroich were involved in patient recruitment and sample and data collection. Robert Seifert and Thomas Decker performed sample analysis and statistical analysis. Thomas Decker, Tobias Dechow, Robert Seifert, Matthias Bichler, Andrea Birtel, and Christoph Nonnenbroich performed data analysis and interpretation. Thomas Decker drafted the manuscript. All the authors participated in the critical revision and validation of the final manuscript.

\section{Data Availability Statement}

All data generated or analyzed during this study are included in this article. Further enquiries can be directed to the corresponding author.

\section{References}

1 Schneeweiss A, Ettl J, Lüftner D, Beckmann MW, Belleville E, Fasching PA, et al. Initial experience with CDK4/6 inhibitor-based therapies compared to antihormone monotherapies in routine clinical use in patients with hormone receptor positive, HER2 negative breast cancer - data from the PRAEGNANT research network for the first 2 years of drug availability in Germany. Breast. 2020; 54:88-95.

2 Goetz MP, Toi M, Campone M, Sohn J, Paluch-Shimon S, Huober J, et al. MONARCH 3: abemaciclib as initial therapy for advanced breast cancer. J Clin Oncol. 2017; 35(32):3638-46.

3 Finn RS, Martin M, Rugo HS, Jones S, Im SA, Gelmon K, et al. Palbociclib and letrozole in advanced breast cancer. N Engl J Med. 2016; 375(20):1925-36

4 Slamon DJ, Neven P, Chia S, Fasching PA, De Laurentiis $\mathrm{M}$, Im SA, et al. Overall survival with ribociclib plus fulvestrant in advanced breast cancer. N Engl J Med. 2020;382(6): 514-24.

5 Im SA, Lu YS, Bardia A, Harbeck N, Colleoni $\mathrm{M}$, Franke F, et al. Overall survival with ribociclib plus endocrine therapy in breast cancer. N Engl J Med. 2019;381(4):307-16.
6 Martin M, Zielinski C, Ruiz-Borrego M, Carrasco E, Turner N, Ciruelos EM, et al. Palbociclib in combination with endocrine therapy versus capecitabine in hormonal receptor-positive, human epidermal growth factor 2-negative, aromatase inhibitor-resistant metastatic breast cancer: a phase III randomised controlled trial-PEARL. Ann Oncol. 2021;32(4):488-99.

7 Park YH, Kim TY, Kim GM, Kang SY, Park IH, Kim JH, et al. Palbociclib plus exemestane with gonadotropin-releasing hormone agonist versus capecitabine in premenopausal women with hormone receptor-positive, HER2-negative metastatic breast cancer (KCSG-BR15-10): a multicentre, open-label, randomised, phase 2 trial. Lancet Oncol. 2019; 20(12):1750-9.

8 Desnoyers A, Nadler MB, Kumar V, Saleh R, Amir E. Comparison of treatment-related adverse events of different cyclin-dependent kinase 4/6 inhibitors in metastatic breast cancer: a network meta-analysis. Cancer Treat Rev. 2020;90:102086.
9 Álvarez-Fernández M, Malumbres M. Mechanisms of sensitivity and resistance to CDK4/6 inhibition. Cancer Cell. 2020;37(4):514-29.

10 Sini V, Cinieri S, Conte P, De Laurentiis M, Leo AD, Tondini C, et al. Endocrine therapy in post-menopausal women with metastatic breast cancer: from literature and guidelines to clinical practice. Crit Rev Oncol Hematol. 2016;100:57-68.

11 Trédan O, Follana P, Moullet I, Cropet C, Trager-Maury S, Dauba J, et al. A phase III trial of exemestane plus bevacizumab maintenance therapy in patients with metastatic breast cancer after first-line taxane and bevacizumab: a GINECO group study. Ann Oncol. 2016;27(6):1020-9.

12 Robertson JFR, Bondarenko IM, Trishkina E, Dvorkin M, Panasci L, Manikhas A, et al. Fulvestrant $500 \mathrm{mg}$ versus anastrozole $1 \mathrm{mg}$ for hormone receptor-positive advanced breast cancer (FALCON): an international, randomised, double-blind, phase 3 trial. Lancet. 2016;388(10063):2997-3005.

13 Cardoso F, Paluch-Shimon S, Senkus E, Curigliano G, Aapro MS, André F, et al. 5th ESOESMO international consensus guidelines for advanced breast cancer (ABC 5). Ann Oncol. 2020;31(12):1623-49. 
14 Masters GA, Temin S, Azzoli CG, Giaccone G, Baker S Jr, Brahmer JR, et al. Systemic therapy for stage IV non-small-cell lung cancer: American Society of Clinical Oncology clinical practice guideline update. J Clin Oncol. 2015;33(30):3488-515.

15 Hegewisch-Becker S, Graeven U, Lerchenmüller CA, Killing B, Depenbusch R, Steffens $\mathrm{CC}$, et al. Maintenance strategies after firstline oxaliplatin plus fluoropyrimidine plus bevacizumab for patients with metastatic colorectal cancer (AIO 0207): a randomised, non-inferiority, open-label, phase 3 trial. Lancet Oncol. 2015;16(13):1355-69.

16 Gligorov J, Doval D, Bines J, Alba E, Cortes P, Pierga JY, et al. Maintenance capecitabine and bevacizumab versus bevacizumab alone after initial first-line bevacizumab and docetaxel for patients with HER2-negative metastatic breast cancer (IMELDA): a randomised, open-label, phase 3 trial. Lancet Oncol. 2014;15(12):1351-60.

17 Seth R, Messersmith H, Kaur V, Kirkwood JM, Kudchadkar R, McQuade JL, et al. Systemic therapy for melanoma: ASCO guideline. J Clin Oncol. 2020;38(33):3947-70.

18 Saussele S, Richter J, Guilhot J, Gruber FX, Hjorth-Hansen H, Almeida A, et al. Discontinuation of tyrosine kinase inhibitor therapy in chronic myeloid leukaemia (EURO-SKI): a prespecified interim analysis of a prospective, multicentre, non-randomised, trial. Lancet Oncol. 2018;19(6):747-57.
19 Palumbo A, Chanan-Khan A, Weisel K, Nooka AK, Masszi T, Beksac M, et al. Daratumumab, bortezomib, and dexamethasone for multiple myeloma. N Engl J Med. 2016; 375(8):754-66.

20 Fountzilas E, Koliou GA, Vozikis A, Rapti V, Nikolakopoulos A, Boutis A, et al. Real-world clinical outcome and toxicity data and economic aspects in patients with advanced breast cancer treated with cyclin-dependent kinase 4/6 (CDK4/6) inhibitors combined with endocrine therapy: the experience of the Hellenic Cooperative Oncology Group. ESMO Open. 2020;5(4):e000774.

21 Cazzaniga ME, Danesi R, Girmenia C, Invernizzi P, Elvevi A, Uguccioni M. Management of toxicities associated with targeted therapies for HR-positive metastatic breast cancer: a multidisciplinary approach is the key to success. Breast Cancer Res Treat. 2019; 176(3):483-94

22 Fogli S, Del Re M, Curigliano G, van Schaik RH, Lancellotti P, Danesi R. Drug-drug interactions in breast cancer patients treated with CDK4/6 inhibitors. Cancer Treat Rev. 2019; $74: 21-8$.

23 Galve-Calvo E, González-Haba E, Gostkorzewicz J, Martínez I, Pérez-Mitru A. Cost-effectiveness analysis of ribociclib versus palbociclib in the first-line treatment of HR+/HER2advanced or metastatic breast cancer in Spain. Clinicoecon Outcomes Res. 2018;10:773-90.
24 Matter-Walstra K, Ruhstaller T, Klingbiel D, Schwenkglenks M, Dedes KJ. Palbociclib as a first-line treatment in oestrogen receptorpositive, HER2-negative, advanced breast cancer not cost-effective with current pricing: a health economic analysis of the Swiss Group for Clinical Cancer Research (SAKK). Breast Cancer Res Treat. 2016;158(1):51-7.

25 Hortobagyi GN, Stemmer SM, Burris HA, Yap YS, Sonke GS, Paluch-Shimon S, et al. Updated results from MONALEESA-2, a phase III trial of first-line ribociclib plus letrozole versus placebo plus letrozole in hormone receptor-positive, HER2-negative advanced breast cancer. Ann Oncol. 2019;30(11):1842.

26 Rugo HS, Finn RS, Diéras V, Ettl J, Lipatov O, Joy AA, et al. Palbociclib plus letrozole as first-line therapy in estrogen receptor-positive/human epidermal growth factor receptor 2-negative advanced breast cancer with extended follow-up. Breast Cancer Res Treat. 2019;174(3):719-29.

27 Sale MJ, Balmanno K, Saxena J, Ozono E, Wojdyla K, McIntyre RE, et al. MEK1/2 inhibitor withdrawal reverses acquired resistance driven by BRAF(V600E) amplification whereas KRAS(G13D) amplification promotes EMT-chemoresistance. Nat Commun. 2019;10(1):2030. 